\title{
Enfermedad granulomatosa hepática
}

Edgar Ortiz-Brizuela, ${ }^{1}$ Daniel Azamar-Llamas, ${ }^{2}$ Jesús Delgado-De la Mora, ${ }^{3}$ Jacobo Iván Guerrero-Castillo, ${ }^{3}$ Braulio Martínez-Benítez ${ }^{3}$ y José Sifuentes-Osornio²

1'Instituto Nacional de Ciencias Médicas y Nutrición "Salvador Zubirán", Departamento de Infectología; 'Instituto Nacional de Ciencias Médicas y Nutrición "Salvador Zubirán", Dirección de Medicina; "3nstituto Nacional de Ciencias Médicas y Nutrición "Salvador Zubirán", Departamento de Patología. Ciudad de México, México

\section{Resumen}

Los granulomas son lesiones circunscritas compuestas principalmente por células mononucleares que surgen en respuesta a estímulos antigénicos pobremente degradables. Se encuentran en 2 a $15 \%$ de las biopsias hepáticas; su hallazgo puede significar desde un fenómeno incidental, hasta la manifestación de una enfermedad sistémica de origen infeccioso, autoinmune o neoplásico. El cuadro clínico suele apuntar a la patología subyacente, sin embargo, la lista de condiciones asociadas es amplia y difiere con base en los antecedentes epidemiológicos y a las características basales del paciente. El elemento de mayor utilidad para su estudio es la historia clínica exhaustiva, con énfasis en viajes recientes, exposición de riesgo y consumo de fármacos o alimentos crudos o exóticos. El análisis histopatológico detallado puede auxiliar en la identificación de la etiología, por ejemplo, la presencia de granulomas epitelioides con necrosis caseosa indica tuberculosis y su ausencia, sarcoidosis; la abundancia de eosinófilos es señal de reacciones farmacológicas o infecciones parasitarias; la presencia de cuerpos extraños puede ser la causa de la enfermedad granulomatosa hepática. En este artículo describimos los aspectos clínico-patológicos básicos de esta enfermedad y proveemos un breve resumen de las etiologías más comunes, principalmente en la región de Latinoamérica.

PALABRAS CLAVE: Enfermedad granulomatosa hepática. Granuloma hepático. Granulomatosis hepática.

\begin{abstract}
Granulomas are circumscribed lesions mainly composed of mononuclear cells that arise in response to poorly degradable antigenic stimuli. They are found in 2-15\% of liver biopsies and the meaning of their finding can range from an incidental phenomenon to the manifestation of a systemic disease of infectious, autoimmune or neoplastic origin. Clinical presentation usually points at the underlying pathology; however, the list of associated conditions is extensive, and differs based on patient epidemiological history and baseline characteristics. The most useful element for their study is a thorough medical history, with an emphasis on recent trips, exposures and consumption of drugs or raw or exotic foods. Detailed histopathological analysis may help identify the etiology. For example, the presence of epithelioid granulomas with caseous necrosis indicates tuberculosis and, its absence, sarcoidosis; eosinophil abundance can be associated with drug reactions or parasitic infections; and the presence of foreign bodies can be the cause of granulomatous liver disease (GLD). In this article, we describe the basic clinical-pathological aspects of GLD, and provide a brief summary of the most common etiologies, with an emphasis on the Latin-American region.
\end{abstract}

KEY WORDS: Granulomatous liver disease. Hepatic granuloma. Hepatic granulomatosis.

Correspondencia:

José Sifuentes-Osornio

E-mail: jose.sifuenteso @ incmnsz.mx
Fecha de recepción: 03-04-2018

Fecha de aceptación: 28-08-2018

DOI: 10.24875/GMM.18004327
Gac Med Mex. 2019;155:266-275

Disponible en PubMed www.gacetamedicademexico.com 


\section{Introducción}

Los granulomas hepáticos (GH) son lesiones circunscritas compuestas principalmente por células mononucleares que surgen en respuesta a estímulos antigénicos pobremente degradables. ${ }^{1}$ Se encuentran en 2 a $15 \%$ de las biopsias hepáticas; su descubrimiento puede representar desde un hallazgo incidental con relevancia clínica limitada hasta la manifestación de una enfermedad sistémica establecida o el hallazgo que lleve a su diagnóstico. ${ }^{2}$ Por lo anterior, su descubrimiento en el contexto clínico apropiado debe llevar al médico a la búsqueda de la causa subyacente. ${ }^{3}$

\section{Fisiopatología}

Los granulomas están compuestos esencialmente por macrófagos y células $\mathrm{T}$. El tipo de respuesta inmune que los desencadena y su aspecto histológico difieren según la naturaleza del agente etiológico y el estado inmunitario del hospedero. ${ }^{1,4,5}$ Por ejemplo, la exposición a agentes débilmente antigénicos resulta en la formación de granulomas persistentes de bajo recambio (por ejemplo, por suturas), también llamados granulomas por cuerpo extraño (Figura 1). Por otro lado, la exposición a estímulos con elevada antigenicidad (por ejemplo, Mycobacterium tuberculosis) da como resultado la activación adicional de respuestas inmunes adaptativas y la formación de granulomas de mayor complejidad (granulomas inmunes) (Figura 2). ${ }^{1,6}$ Tanto el sistema inmune innato como el adaptativo se encuentran involucrados en su formación y mantenimiento. ${ }^{7}$ En el caso de Mycobacterium tuberculosis, su reconocimiento por medio de diversos receptores macrofágicos (por ejemplo, receptor del complemento 4), entre otros factores, permite la activación de las células T cooperadoras hacia células Th1, Th2, Th17 o T-reguladoras, así como el reclutamiento de otras células inflamatorias (macrófagos, neutrófilos, células B). Lo anterior resulta en la formación de la estructura del granuloma: un centro compuesto por macrófagos intercalados con neutrófilos, rodeado por un anillo de linfocitos $B$ y T. ${ }^{8}$ El balance en la síntesis de diversas citocinas con actividad pro (IFN- $\gamma$, TNF- $\alpha$, IL-12) y antiinflamatoria (IL-4 e IL-10) en esta estructura, junto a otros determinantes, define las características y desenlaces del granuloma. ${ }^{9,10}$ Clásicamente, el predominio de alguno de estos fenotipos inmunes se ha relacionado con

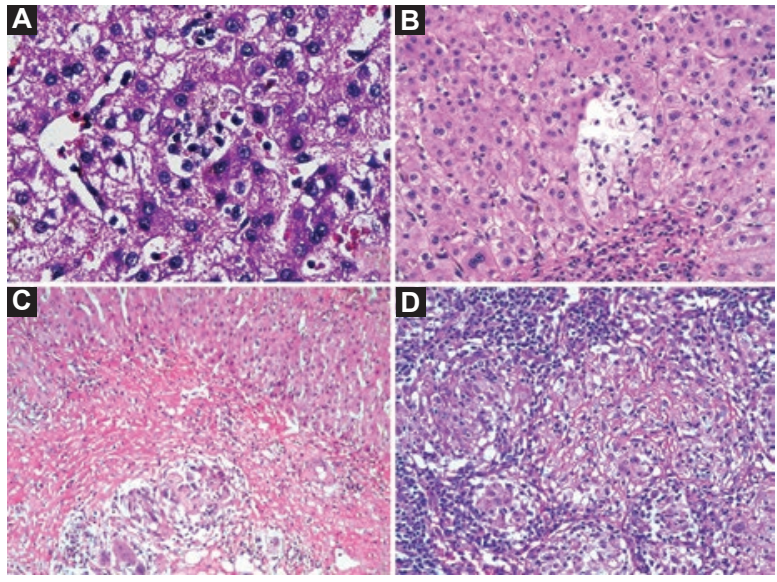

Figura 1. A) Granuloma epitelioide sin necrosis en localización lobulillar en paciente con colangitis biliar primaria (400x). B) Macrófagos espumosos en lobulillo en paciente con colangitis esclerosante primaria (400x). C) Hepatitis granulomatosa con células gigantes multinucleadas de tipo cuerpo extraño posterior a procedimiento quirúrgico (100x). D) Hepatitis granulomatosa en sarcoidosis, los granulomas son pequeños, confluentes y sin necrosis caseosa (100x). La tinción empleada fue hematoxilina-eosina.
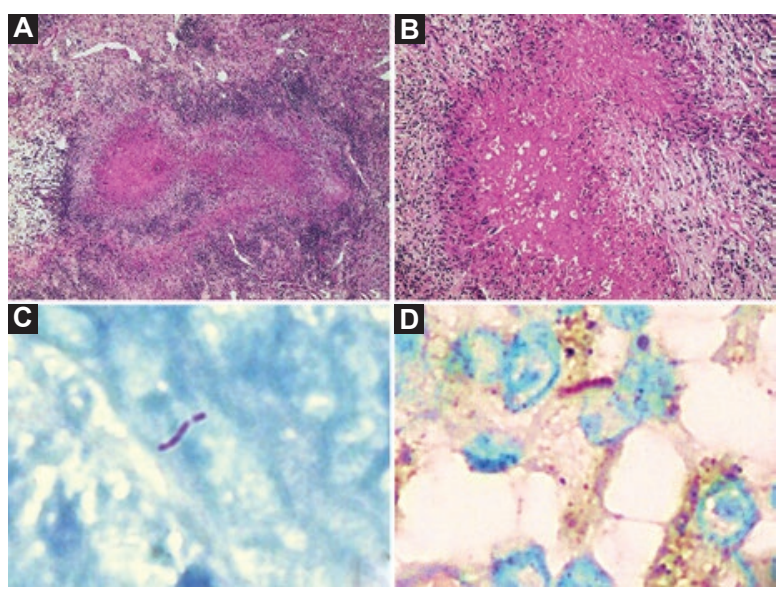

Figura 2. A) Granuloma hepático con necrosis caseosa central (hematoxilina-eosina 40x). B) Granuloma hepático donde se identifican macrófagos con formación de empalizadas alrededor de la necrosis caseosa (hematoxilina-eosina 100x). C) La tinción de ZiehlNeelsen fue positiva para micobacterias. D) Tinción de Ziehl-Neelsen e inmunohistoquímica para Mycobacterium tuberculosis (1000x).

etiologías específicas: Th1 con tuberculosis y sarcoidosis y Th2 con infecciones parasitarias como la esquistosomiasis. ${ }^{11}$

\section{Histopatología}

Diversos componentes morfológicos auxilian en la identificación de la etiología de la enfermedad granulomatosa hepática (EGH), como su localización dentro del parénquima hepático (por ejemplo, portal en colangitis biliar primaria o periportal en sarcoidosis), el tipo de granuloma o la presencia de algunos componentes 
en particular (eosinófilos en reacciones farmacológicas o infecciones parasitarias o cuerpos extraños como bario, suturas, talco).2,7 Sin embargo, es importante hacer énfasis en que la mayoría de estos hallazgos no son específicos de alguna causa. 2,7,12 A grandes rasgos, se han descrito las siguientes clases morfológicas de $\mathrm{GH}: 6,12$

- Granulomas epitelioides. Son colecciones de céIulas epitelioides cuya función es sustancialmente secretora. ${ }^{6}$ Se caracterizan por bordes delimitados del parénquima hepático. Pueden encontrarse asociados con diversos tipos de necrosis (por ejemplo, caseosa), comúnmente de etiología infecciosa, principalmente tuberculosis, pero también histoplasmosis y candidiasis, entre otras (Figuras 2). También se encuentran en patologías como la sarcoidosis o la colangitis biliar primaria, en cuyos casos la necrosis suele estar ausente (Figura 1). 2,6

- Granulomas supurativos (granulomas con microabscesos centrales). Están compuestos por un absceso central rodeado de un anillo de células epitelioides o células espumosas. Se han relacionado con infecciones fúngicas (por ejemplo, histoplasmosis, candidiasis), actinomicosis e infecciones por Nocardia spp. ${ }^{6}$

- Microgranulomas. Compuestos por tres a siete macrófagos mezclados con hepatocitos apoptóticos y otras células inflamatorias. ${ }^{6}$ Es un patrón muy inespecífico que puede surgir ante cualquier enfermedad inflamatoria hepática crónica, por ejemplo, por reacciones adversas a fármacos (betalactámicos, sulfonamidas, diazepam, alopurinol, diclofenaco). . $^{6,12,13}$

- Agregados de macrófagos espumosos. Están integrados por células espumosas con una respuesta inflamatoria adicional escasa. ${ }^{6}$ Se observan especialmente en enfermedades infecciosas en pacientes inmunocomprometidos (portadores del virus de inmunodeficiencia humana, VIH). Ejemplos son las infecciones por el complejo Mycobacterium avium, la lepra lepromatosa, la histoplasmosis y la leishmaniasis (Figura 3). ${ }^{12}$

- Granulomas con anillo de fibrina. Sus rasgos distintivos son una vacuola lipídica central y un anillo periférico de fibrina (Figura 4). Se han descrito principalmente con la fiebre $Q$, sin embargo, existen reportes con diversas etiologías (citomegalovirus, leishmaniasis, virus de hepatitis A y C [VHC], toxoplasmosis, arteritis de células gigantes, alopurinol, linfomas)..$^{2,6}$

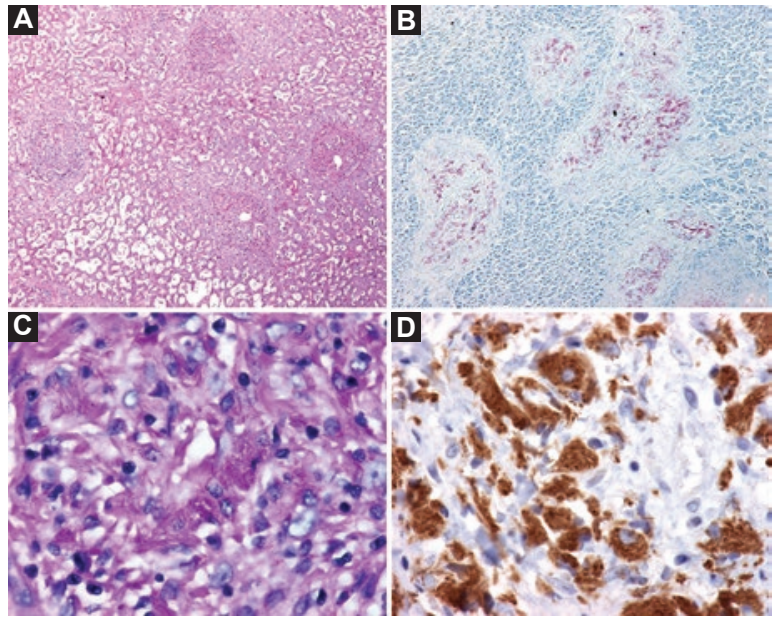

Figura 3. A) Granulomas con macrófagos espumosos en parénquima hepático (hematoxilina-eosina 40x). B) Granulomas con macrófagos espumosos y abundantes micobacterias (Ziehl-Neelsen 40x). C) Macrófagos espumosos con abundantes micobacterias (ácido peryódico de Schiff 400x). D) Inmunohistoquímica positiva en micobacterias dentro de macrófagos espumosos (400x).

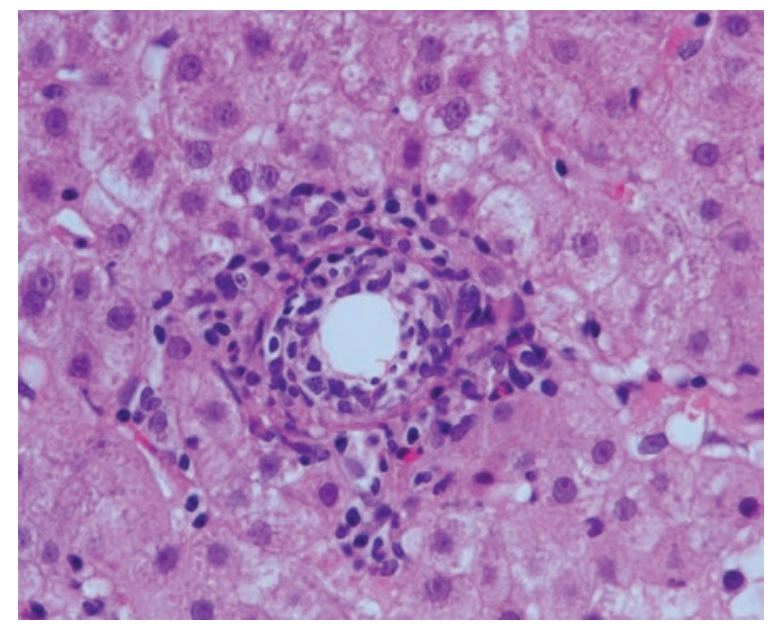

Figura 4. Granuloma con anillo de fibrina en un paciente con fiebre $Q$ (hematoxilina-eosina 100x).

- Lipogranulomas. Contienen una o múltiples vacuolas lipídicas centrales rodeadas de histiocitos y macrófagos. ${ }^{6}$ Generalmente se encuentran en la zona hepática 3 , frecuentemente asociados con la esteatosis hepática, la ingestión de aceite mineral o con la infección por VHC. 2,6,12,14

\section{Cuadro clínico}

Los GH son indolentes, no obstante, entre 70 y $80 \%$ de los pacientes presenta alguna sintomatología secundaria a la patología subyacente. ${ }^{15,16}$ En consecuencia, las características clínicas varían acorde con la epidemiología de la región geográfica evaluada. ${ }^{16,17}$ En los estudios realizados en países en desarrollo, 
Tabla 1. Etiologías asociadas con la enfermedad granulomatosa hepática

\begin{tabular}{|c|c|c|c|c|}
\hline \multirow[t]{5}{*}{ Infecciosas } & $\begin{array}{l}\text { Micobacterias } \\
\text { Mycobacterium tuberculosis }\end{array}$ & Mycobacterium bovis & Mycobacterium avium-intracellulare & Mycobacterium leprae \\
\hline & $\begin{array}{l}\text { Bacterias } \\
\text { Brucella spp. } \\
\text { Actinomyces spp. } \\
\text { Bartonella henselae }\end{array}$ & $\begin{array}{l}\text { Salmonella spp. } \\
\text { Coxiella burnetii } \\
\text { Rhodococcus equi }\end{array}$ & $\begin{array}{l}\text { Treponema pallidum } \\
\text { Rickettsia spp. } \\
\text { Francisella tularensis }\end{array}$ & $\begin{array}{l}\text { Chlamydia spp. } \\
\text { Listeria monocytogenes }\end{array}$ \\
\hline & $\begin{array}{l}\text { Parásitos } \\
\text { Schistosoma spp. } \\
\text { Strongyloides stercoralis } \\
\text { Toxoplasma gondii }\end{array}$ & $\begin{array}{l}\text { Equinococcus spp. } \\
\text { Giardia lamblia }\end{array}$ & $\begin{array}{l}\text { Enterobius vermicularis } \\
\text { Leishmania sp. }\end{array}$ & $\begin{array}{l}\text { Fasciola hepatica } \\
\text { Entamoeba spp. }\end{array}$ \\
\hline & $\begin{array}{l}\text { Virales } \\
\text { Hepatitis B, C }\end{array}$ & Virus Epstein-Barr & & Citomegalovirus \\
\hline & $\begin{array}{l}\text { Fúngicas } \\
\text { Histoplasma capsulatum } \\
\text { Candida spp. } \\
\text { Mucor spp. }\end{array}$ & $\begin{array}{l}\text { Coccidioides spp. } \\
\text { Cryptococcus spp. }\end{array}$ & $\begin{array}{l}\text { Paracoccidiodes brasiliensis } \\
\text { Aspergillus spp. }\end{array}$ & $\begin{array}{l}\text { Blastomyces dermatitidis } \\
\text { Pneumocystis jirovecii }\end{array}$ \\
\hline Autoinmunidad & Colangitis biliar primaria & Vasculitis sistémicas & Lupus eritematoso sistémico & \\
\hline Cuerpos extraños & Aceite mineral & Almidón & Silicón & \\
\hline Neoplasias & Linfoma de Hodgkin & Linfoma no-Hodgkin & & \\
\hline Otras causas & Sarcoidosis & Reacciones a fármacos & & \\
\hline
\end{tabular}

donde la causa más frecuentemente reportada es tuberculosis, los síntomas más comunes son fiebre, pérdida de peso y fatiga. ${ }^{16,18}$ Por el contrario, en países desarrollados, los síntomas como el prurito y la fatiga podrían ser más frecuentes debido a la mayor prevalencia de colangitis biliar primaria y sarcoidosis. ${ }^{7}$ Ocasionalmente la EGH per se puede provocar dolor abdominal, hepatomegalia, hipertensión portal 0 colestasis. ${ }^{16}$

Hasta $63 \%$ de los casos de EGH puede presentar alteraciones de las pruebas de funcionamiento hepático como hiperfosfatasia, hiperbilirrubinemia e hipertransaminasemia. ${ }^{15,16}$ En pacientes con sarcoidosis, colangitis biliar primaria y tuberculosis se ha descrito predominantemente hiperfosfatasia. Por otro lado, en patologías asociadas con un menor daño de los canalículos biliares (por ejemplo, VHC) se puede observar con mayor frecuencia hipertransaminasemia. ${ }^{2,7,17}$ Los estudios de imagen pueden ser de utilidad; mediante ellos se pueden encontrar hepatomegalia 0 lesiones nodulares secundarias a la presencia de los $\mathrm{GH}^{2,17}$

\section{Diagnóstico diferencial}

La lista de patologías asociadas con EGH es extensa y el diagnóstico diferencial difiere según la epidemiología local, las características del paciente y el historial de exposiciones (Tabla 1). ${ }^{3}$ El conjunto del cuadro clínico, estudios paraclínicos y hallazgos histopatológicos es el que posee mayor utilidad para la identificación de la etiología. En la evaluación médica es preciso un interrogatorio exhaustivo (con énfasis en averiguar viajes recientes, exposiciones de riesgo, ingesta de fármacos o consumo de alimentos crudos o exóticos) y un examen físico detallado (Algoritmo 1). Los estudios paraclínicos deberán solicitarse razonadamente con base en el juicio clínico. En caso de no apreciar una etiología evidente, se sugiere que la búsqueda sea dirigida a las causas más comunes en la población en cuestión. ${ }^{15}$ Las etiologías más frecuentemente reportadas en los países desarrollados son sarcoidosis, enfermedades autoinmunes, tuberculosis y EGH idiopática. ${ }^{3,15,19-23}$ Contrariamente, la frecuencia de enfermedades autoinmunes es menor en los países en desarrollo (por ejemplo, Arabia Saudita, Irán, India y Turquía), donde las principales causas son las enfermedades infecciosas (tuberculosis y esquistosomiasis). $6,16,18,24-26$

En México y el resto de América Latina, en ausencia de datos epidemiológicos de la EGH, un abordaje inicial sensato en pacientes cuyo cuadro clínico no sea sugerente de patología específica podría incluir una radiografía de tórax, cultivos de muestras hepáticas en medios especiales para bacterias, micobacterias y hongos y un análisis histopatológico detallado 
con el empleo de tinciones especiales (por ejemplo, Fite Faraco, Ziehl-Neelsen, ácido peryódico de Schiff, Grocott, Giemsa). Pruebas complementarias de utilidad son algunas serologías (virus de hepatitis $B$, VHC, VIH, sífilis, fiebre Q, Brucella spp, y anticuerpos antimitocondriales), la prueba de la tuberculina cutánea, los ensayos funcionales de liberación de interferón gamma y pruebas moleculares como la PCR para búsqueda de Mycobacterium tuberculosis. ${ }^{2,15}$

\section{Etiología}

A pesar de un abordaje exhaustivo, hasta $50 \%$ de los casos puede permanecer sin diagnóstico etiológico (EGH idiopática); ${ }^{7,15} 15 \%$ de estos enfermos puede desarrollar síntomas durante el seguimiento, lo que obliga a vigilancia clínica durante un tiempo suficiente (12 a 24 meses). Una proporción importante de los pacientes con EGH idiopática puede permanecer asintomática y con un buen pronóstico a largo plazo. ${ }^{3,15,19,21}$ Sin embargo, existen reportes de casos de síndromes febriles persistentes asociados con EGH idiopática en los que se ha documentado respuestas favorables con glucocorticoides o metotrexato. ${ }^{27} \mathrm{~A}$ continuación, se hace una breve descripción de sus principales etiologías.

\section{Enfermedades autoinmunes}

\section{ColangITIS BILIAR PRIMARIA}

La colangitis biliar primaria es una enfermedad hepática autoinmune que afecta predominantemente a mujeres de mediana edad. Condiciona destrucción de los canalículos biliares intrahepáticos y fibrosis hepática progresiva. ${ }^{28}$ Es la causa más frecuente de EGH en algunos países de occidente (Alemania, Grecia, Irlanda, Escocia e Italia) y la segunda en Estados Unidos, responsable de 20 a $68 \%$ y $22 \%$ de los casos, respectivamente..$^{3,19}$ Los datos en América Latina se encuentran limitados a series de casos. ${ }^{29}$ Se manifiesta por un síndrome colestásico en ausencia de obstrucción de la vía biliar extrahepática. Su diagnóstico requiere dos de tres de los siguientes: anticuerpos antimitocondriales positivos, hiperfosfatasia o evidencia histológica de colangitis biliar primaria (colangitis destructiva no supurativa y destrucción de los conductos biliares interlobulillares). Su imagen histopatológica característica es la lesión florida del conducto biliar (degeneración del epitelio ductal biliar con obliteración ductal focal y formación de granulomas con distribución portal predominante). ${ }^{2,7,30}$ Actualmente, la única terapia aprobada es el ácido ursodesoxicólico. ${ }^{28}$

\section{Enfermedades infecciosas}

Son responsables de EGH en 55 a $75 \%$ de los casos en Arabia Saudita, Irán e India. ${ }^{16,18,26}$ La EGH puede ser resultado de una afección hepática primaria 0 de un padecimiento generalizado. Las causas más comunes son tuberculosis, algunas zoonosis (por ejemplo, fiebre $Q$, brucelosis), infecciones oportunistas en pacientes portadores de VIH (por ejemplo, Mycobacterium avium), infecciones fúngicas diseminadas (por ejemplo, histoplasmosis) e infección por VHC. ${ }^{12}$ Los granulomas hepáticos característicos de las etiologías infecciosas son granulomas con necrosis asociada, por lo que su presencia debe siempre orientar hacia su búsqueda. ${ }^{31}$

\section{TUBERCULOSIS}

La tuberculosis es la causa infecciosa más común de EGH en la India (55\%), Arabia Saudita (42.6\%) e Irán $(51.4 \%)^{16,18,26}$ En América Latina no existen datos acerca de la prevalencia de tuberculosis en la $E G H$, no obstante, la incidencia de tuberculosis en esta región geográfica se reportó en 27 casos por 100000 habitantes en 2017. ${ }^{32}$ Por lo general es resultado de la diseminación hematógena posterior a su adquisición por vía pulmonar o gastrointestinal (50 a $80 \%$ ). La afección limitada al hígado es una manifestación poco común de la infección, en cuyo caso debe sospecharse Mycobacterium bovis debido a su mayor involucro en sitios extrapulmonares..$^{12,31,33,34}$ El cuadro clínico se encuentra dominado por los síntomas de afección extrahepática y el involucro hepático por sí mismo se ha asociado con dolor abdominal inespecífico o en el cuadrante superior derecho (65 a $87 \%$ de los casos). ${ }^{33}$ Existen reportes de casos de su presentación como colecistitis aguda o síndrome colestásico. ${ }^{33,35}$ Los estudios de imagen pueden mostrar hepatomegalia, abscesos, lesiones sólidas focales que semejan tumores hepáticos e, incluso, estenosis de los conductos biliares. ${ }^{36}$

Los hallazgos histopatológicos característicos son los granulomas epitelioides de focos múltiples con caseificación central y necrosis fibrinoide de distribución periportal predominante, sin embargo, la ausencia de necrosis no descarta esta etiología. 12,33 Los granulomas pueden fusionarse y formar 
tuberculomas, nódulos con un diámetro de 1 a $4 \mathrm{~cm}$, que a su vez pueden presentar calcificaciones características. ${ }^{33}$ El diagnóstico de la tuberculosis hepática se basa en la demostración de bacilos ácido-alcohol resistentes en frotis, cultivos o por PCR (sensibilidad y especificidad de $88 \%$ y $100 \%$, respectivamente). ${ }^{33,37,38} \mathrm{El}$ tratamiento de la tuberculosis hepática no difiere del requerido para cualquier afección extrapulmonar por tuberculosis. ${ }^{33,39}$

\section{INFECCIONES OPORTUNISTAS EN PACIENTES PORTADORES DE VIH}

Las infecciones oportunistas en los pacientes portadores del VIH presentan variación regional y según el grado de disfunción inmune. Al inicio de la pandemia, las series mexicanas reportaron que la mayor parte de las infecciones oportunistas fueron secundarias a Candida spp, Pneumocystis jirovecii, Cryptosporidium spp., Mycobacterium tuberculosis, citomegalovirus, Histoplasma spp. y Mycobacterium avium. ${ }^{40}$ No obstante, desde el advenimiento de la terapia antirretroviral (TAR) se ha observado importante reducción en su incidencia. Las etiologías de la EGH en estos enfermos también han experimentado esta transición epidemiológica. ${ }^{41}$

En un estudio realizado en Estados Unidos en la etapa preTAR se encontró que las causas más frecuentes de afección hepática fueron Mycobacterium avium/Mycobacterium avium intracellulare (17.6\%), las hepatitis virales crónicas (12\%) y la tuberculosis $(2.6 \%) .^{42}$ En México se encontró una prevalencia de EGH de $29 \%$ de las biopsias hepáticas. Las etiologías más comunes de afección hepática fueron Mycobacterium tuberculosis (26.6\%), Histoplasma capsulatum (20\%), citomegalovirus (13.3\%) y Mycobacterium avium/Mycobacterium avium intracellulare (11\%). Sin embargo, en el análisis restringido a casos de EGH, la etiología más común fue Mycobacterium avium/ Mycobacterium avium intracellulare..$^{43}$

Respecto a la etapa posTAR, las principales causas de enfermedad hepática son las coinfecciones VHC/ $\mathrm{VIH}$, hepatitis $\mathrm{B} / \mathrm{VIH}$ y la hepatotoxicidad por la TAR. ${ }^{41,44}$ Debido a que el diagnóstico e inicio de TAR tardíos siguen siendo escenarios comunes en América Latina, es esperable la presencia de casos de EGH con características de ambas etapas epidemiológicas (pre y posTAR). ${ }^{45}$ Por lo tanto, es primordial la evaluación del estado de disfunción inmune del enfermo al establecer las causas potenciales de EGH.

\section{Zoonosis}

Dos ejemplos de zoonosis que se han asociado con $E G H$ son la fiebre $Q$ y la brucelosis, ambas presentes en México y en otras regiones de América Latina como Argentina, Brasil, Colombia, Costa Rica y Ecuador. ${ }^{46-51}$ La fiebre $Q$ es una enfermedad causada por Coxiella burnetii (bacteria intracelular); el principal factor de riesgo es la exposición a animales de granja (ganado bovino, ovino o caprino), a través de la inhalación de aerosoles de suelos contaminados por heces, orina 0 a placentas de animales infectados. Frecuentemente el diagnóstico se hace en pacientes sin historia de contacto directo con este tipo de animales; se cree que esto puede deberse a la viabilidad de las esporas de Coxiella burnetii y a su capacidad de viajar largas distancias por el viento. ${ }^{52}$ Los granulomas característicos de esta enfermedad son aquellos con anillo de fibrina (Figura 4). ${ }^{12,52}$ Clínicamente se manifiesta por cuadros autolimitados de fiebre, cefalea, neumonía y hepatitis, con el desarrollo de cuadros crónicos en menos de $5 \%$ de los casos (por ejemplo, endocarditis infecciosa, osteomielitis, hepatitis). Su diagnóstico se basa en la PCR de sangre y la serología, en tanto que el tratamiento de elección es la doxiciclina. ${ }^{53}$

La brucelosis es una enfermedad granulomatosa causada por Brucella spp.; su vía de adquisición es a través del contacto directo con ganado (principalmente vacuno, caprino o porcino) o por el consumo de productos lácteos no pasteurizados..$^{54}$ La brucelosis puede afectar cualquier órgano; el cuadro clínico varía desde un cuadro febril agudo poco específico (por ejemplo, fiebre, cefalea, poliartralgias, adenopatías, hepatoesplenomegalia) hasta una afección crónica de predominio osteoarticular y neuropsiquiátrica. ${ }^{54} \mathrm{La}$ afectación hepática puede ocurrir hasta en $50 \%$ de los casos; los hallazgos histopatológicos son los granulomas epitelioides no caseificantes o microgranulomas. ${ }^{55}$ Se debe considerar esta etiología en pacientes con fiebre sin otra causa evidente; su diagnóstico se hace a través de su aislamiento en el cultivo, PCR o serología. ${ }^{12,54}$

\section{Infecciones fúngicas}

Las infecciones fúngicas se observan principalmente como infecciones diseminadas en pacientes inmunocomprometidos (por ejemplo, neutropenia). Destacan la candidiasis hepatoesplénica, la aspergilosis invasiva, la criptococosis, y algunas micosis endémicas (histoplasmosis, coccidiodomicosis, paracoccidiodomicosis $y$ 
blastomicosis). ${ }^{2,56-60}$ La coccidiodomicosis es altamente prevalente en las regiones secas y con temperaturas elevadas; Coccidioides immitis se ha descrito predominantemente en el Valle de San Joaquín (California) y Coccidioides posadasii en Arizona, Texas, México, Centroamérica (Guatemala, Honduras) y Sudamérica (Argentina, Colombia, Paraguay y Venezuela). ${ }^{61}$ Su forma clínica de presentación más común es la afección pulmonar y en algunos casos puede observarse diseminación. ${ }^{62}$

La histoplasmosis es la micosis endémica más frecuente en Centroamérica, también presente en Argentina, Brasil, Colombia, Ecuador, Uruguay y Venezuela. ${ }^{61}$ Su vía de entrada es respiratoria y los granulomas hepáticos asociados se caracterizan por infiltrados linfohistiocíticos con hiperplasia sinusoidal de las células de Kupffer, acompañados de levaduras pequeñas ( 3 a 4 de $\mu \mathrm{m}$ de diámetro) ovoides únicas o en gemación, de base estrecha, detectables por medio de la tinción con PAS. ${ }^{12,63-65}$ Elementos diagnósticos útiles por su especificidad y valor predictivo negativo elevados en formas diseminadas son la PCR en sangre o tejido y la detección de sus antígenos en orina; el diagnóstico definitivo es mediante su aislamiento. ${ }^{66}$

\section{Infección por VHC y VHB}

Las infecciones por los virus de las hepatitis $C$ y $B$ se han asociado con EGH en ausencia de otras causas atribuibles. ${ }^{67-69}$ En los pacientes con infección crónica por VHC sometidos a biopsia hepática se ha reportado una prevalencia de $1.3 \%$, principalmente en pacientes con antecedente de tratamiento con interferón. ${ }^{67,70} \mathrm{De}$ bido a la disponibilidad de nuevas técnicas no invasivas para la estadificación de la fibrosis en estos pacientes, así como el advenimiento de tratamiento antivirales más efectivos, es esperable que en el futuro encontremos esporádicamente estas etiologías de EGH. ${ }^{44}$

En América Latina, la única etiología reportada de esquistosomiasis es Schistosoma mansoni (Brasil, Venezuela, Suriname, Puerto Rico y el Caribe) $\cdot{ }^{71}$ Los casos en México suelen estar asociados con inmigrantes. ${ }^{72,73}$ El principal factor asociado es la exposición a agua contaminada por caracoles del género Biomphalaria. Es la principal causa de hipertensión portal en el mundo; los parásitos invaden las venas mesentéricas o la vena porta, donde depositan sus huevecillos, estos producen una reacción de hipersensibilidad, lo que resulta en fibrosis y enfermedad hepatobiliar obstructiva. En fases crónicas de la enfermedad suele aparecer una reacción granulomatosa alrededor de los huevecillos acompañada de zonas de fibrosis con abundantes eosinófilos. ${ }^{12}$

\section{Neoplasias}

Existen reportes de neoplasias como el linfoma de Hodgkin, no-Hodgkin y el carcinoma renal de células claras, cuya presentación inicial son los GH epitelioides no caseificantes. ${ }^{2,21,31,74}$ En áreas endémicas de tuberculosis, como México, se ha descrito la concurrencia de ambas etiologías, por ello es recomendable que esta se busque en este grupo de enfermos en países con elevada endemicidad de tuberculosis. ${ }^{75}$

\section{Otras etiologías}

La sarcoidosis es una enfermedad con baja incidencia en América Latina, ${ }^{76,77} \sin$ embargo, representa una de las principales causas de EGH en Estados Unidos y en algunas regiones de Europa. ${ }^{2,15,19}$ La frecuencia de afección hepática varía de $6 \%$ en estudios de base poblacional y hasta $70 \%$ en estudios de autopsias. ${ }^{2,17,78}$ La afectación clínica (dolor abdominal, hepatomegalia) es poco frecuente (25 a $40 \%$ ), por lo que la forma más común de detección es el descubrimiento de la alteración del funcionamiento hepático (hiperfosfatasia, elevación de la gamma glutamil transpeptidasa e hipertransaminasemia). ${ }^{17} \mathrm{~A}$ su vez, se pueden observar anomalías en los estudios de imagen en $50 \%$ de los pacientes (por ejemplo, lesiones nodulares hipodensas o hepatomegalia). ${ }^{17}$ Los granulomas característicos son epitelioides no caseificantes con afección periportal predominante; no obstante, para su diagnóstico es primordial la exclusión de otras causas principalmente infecciosas. ${ }^{79}$ La sarcoidosis hepática rara vez progresa a cirrosis $(6$ a $24 \%$ ), por lo que el tratamiento se reserva para pacientes con afección grave..$^{17,80}$

La lista de fármacos asociados a EGH es amplia y comprende más de 60 drogas; destacan algunos antibióticos (por ejemplo, sulfas, isoniazida, nitrofurantoína, norfloxacino), anticonvulsivos (por ejemplo, fenitoína, carbamazepina), alopurinol y paracetamol. ${ }^{2,19} \mathrm{La}$ EGH por fármacos puede tener un inicio agudo o crónico y, al igual que el resto de las etiologías, puede presentar un curso indolente detectado solo por un patrón de daño hepatocelular o colestásico. ${ }^{81}$ Histopatológicamente con frecuencia se encuentran microglanulomas y, ocasionalmente, puede haber infiltración 
por eosinófilos, lo que orienta el diagnóstico, ${ }^{82}$ que se lleva a cabo por exclusión.

\section{Conclusiones}

El espectro clínico de la EGH es muy extenso, comprende desde un hallazgo incidental con poca relevancia clínica hasta un dato pivote de una enfermedad generalizada. Estos distintos escenarios deben cotejarse con la sintomatología, los hallazgos histopatológicos, los antecedentes epidemiológicos y los estudios paraclínicos complementarios. Elementos útiles en el diagnóstico son una historia clínica exhaustiva, la revisión profunda de la histopatología, con un bajo umbral para la realización de tinciones especiales, pruebas microbiológicas y moleculares. En México se necesitan más estudios de la EGH para conocer las etiologías más frecuentes.

\section{Bibliografía}

1. Chensue SW. Chemokines in innate and adaptive granuloma formation. Front Immunol. 2013:4:43

2. Coash M, Forouhar F, Wu CH, Wu GY. Granulomatous liver diseases: a review. J Formos Med Assoc. 2012;111:3-13

3. Drebber U, Kasper HU, Ratering J, Wedemeyer I, Schirmacher P, Dienes HP, et al. Hepatic granulomas: histological and molecular pathological approach to differential diagnosis: a study of 442 cases. Liver Int. 2008;28:828-834

4. Peddireddy V, Doddam SN, Ahmed N. Mycobacterial Dormancy systems and host responses in tuberculosis. Front Immunol. 2017;8:84.

5. Shaker Y, Samy N, Ashour E. Hepatobiliary schistosomiasis. J Clin Transl Hepatol. 2014:2:212-216.

6. Saxena R. Hepatic granulomas: differential diagnosis. En: Practical hepatic pathology: a diagnostic approach. EE. UU.: Saunders/Elsevier; 2011.

7. Almadi MA, Aljebreen AM, Sanai FM, Marcus V, Almeghaiseeb ES, Ghosh S. New insights into gastrointestinal and hepatic granulomatous disorders. Nat Rev Gastroenterol Hepatol. 2011;8:455-466.

8. Scriba TJ, Coussens AK, Fletcher HA. Human immunology of tubercuIosis. Microbiol Spectr. 2016;4.

9. Dorhoi A, Reece ST, Kaufmann SH. For better or for worse: the immune response against Mycobacterium tuberculosis balances pathology and protection. Immunol Rev. 2011;240:235-251.

10. Guirado E, Schlesinger LS. Modeling the mycobacterium tuberculosis granuloma: the critical battlefield in host immunity and disease. Front Immunol. 2013;4:98.

11. Lagana SM, Moreira RK, Lefkowitch JH. Hepatic granulomas: pathogenesis and differential diagnosis. Clin Liver Dis. 2010;14:605-617.

12. Lamps LW. Hepatic granulomas: a review with emphasis on infectious causes. Arch Pathol Lab Med. 2015;139:867-875.

13. Ramachandran R, Kakar S. Histological patterns in drug-induced liver disease. J Clin Pathol. 2009;62:481-492.

14. Zhu H, Bodenheimer HC, Clain DJ, Min AD, Theise ND. Hepatic lipogranulomas in patients with chronic liver disease: association with hepatitis $C$ and fatty liver disease. World J Gastroenterol. 2010;16:5065-5069.

15. Sartin JS, Walker RC. Granulomatous hepatitis: a retrospective review of 88 cases at the Mayo Clinic. Mayo Clin Proc. 1991;66:914-918.

16. Sanai FM, Ashraf S, Abdo AA, Satti MB, Batwa F, Al-Husseini H, et al. Hepatic granuloma: decreasing trend in a high-incidence area. Liver Int. 2008:28:1402-1407.

17. Ungprasert $P$, Crowson CS, Simonetto DA, Matteson EL. Clinical characteristics and outcome of hepatic sarcoidosis: a population-based study 1976-2013. Am J Gastroenterol. 2017;112:1556-1563.

18. Sabharwal BD, Malhotra N, Garg R, Malhotra V. Granulomatous hepatitis: a retrospective study. Indian J Pathol Microbiol. 1995;38:413-416.

19. Wainwright H. Hepatic granulomas. Eur J Gastroenterol Hepatol. 2007:19:93-95.

20. Dourakis SP, Saramadou R, Alexopoulou A, Kafiri G, Deutsch M, Koskinas J, et al. Hepatic granulomas: a 6-year experience in a single center in Greece. Eur J Gastroenterol Hepatol. 2007;19:101-104.
21. Gaya DR, Thorburn D, Oien KA, Morris AJ, Stanley AJ. Hepatic granulomas: a 10 year single centre experience. J Clin Pathol. 2003;56:850-853.

22. Guglielmi V, Manghisi OG, Pirrelli M, Caruso ML. [Granulomatous hepatitis in a hospital population in southern Italy]. Pathologica 1994;86:271-8.

23. McCluggage WG, Sloan JM. Hepatic granulomas in Northern Ireland: a thirteen year review. Histopathology. 1994;25:219-228.

24. Satti MB, Al-Freihi H, Ibrahim EM, Abu-Melha A, Al-Ghassab G, Al-Idrissi HY, et al. Hepatic granuloma in Saudi Arabia: a clinicopathological study of 59 cases. Am J Gastroenterol. 1990;85:669-674.

25. Mert A, Ozaras R, Bilir M, Tahan V, Cetinkaya A, Yirmibescik S, et al. The etiology of hepatic granulomas. J Clin Gastroenterol 2001;32:275-6.

26. Geramizadeh B, Jahangiri R, Moradi E. Causes of hepatic granuloma: a 12-year single center experience from southern Iran. Arch Iran Med. 2011;14:288-289.

27. Knox TA, Kaplan MM, Gelfand JA, Wolff SM. Methotrexate treatment of idiopathic granulomatous hepatitis. Ann Intern Med. 1995;122:592-595.

28. Carey EJ, Ali AH, Lindor KD. Primary biliary cirrhosis. Lancet. 2015; 386:1565-1575.

29. Rodríguez-Lugo DA, Coronado-Tovar JJ, Solano-Villamarin GA, Otero-Regino W. Primary biliary cholangitis. Part 1. State of the art, epidemiology, physiopathology and clinical manifestations. Rev Gastroenterol Peru. 2017:37(4):357-364.

30. Aguilar-Nájera O, Velasco-Zamora JA, Torre A. Overlap syndromes of autoimmune hepatitis: diagnosis and treatment. Rev Gastroenterol Mex. 2015;80:150-159

31. Turhan N, Kurt M, Ozderin YO, Kurt OK. Hepatic granulomas: a clinicopathologic analysis of 86 cases. Pathol Res Pract. 2011;207:359-365.

32. Global tuberculosis report 2017. Suiza: World Health Organization; 2017.

33. Chaudhary P. Hepatobiliary tuberculosis. Ann Gastroenterol. 2014; 27:207-211

34. Torres-González P, Cervera-Hernández ME, Martínez-Gamboa A, García-García L, Cruz-Hervert LP, Bobadilla-Del Valle M, et al. Human tuberculosis caused by mycobacterium bovis: a retrospective comparison with mycobacterium tuberculosis in a Mexican tertiary care centre, 20002015. BMC Infect Dis. 2016;16(1):657.

35. Kok KY, Yapp SK. Tuberculosis of the bile duct: a rare cause of obstructive jaundice. J Clin Gastroenterol. 1999;29:161-164.

36. Karaosmanoglu AD, Onur MR, Sahani DV, Tabari A, Karcaaltincaba M. Hepatobiliary tuberculosis: imaging findings. AJR Am J Roentgenol. 2016;207:694-704

37. Lewinsohn DM, Leonard MK, LoBue PA, Cohn DL, Daley CL, Desmond E, et al. Official American Thoracic Society/Infectious Diseases Society of America/Centers for Disease Control and Prevention Clinical Practice Guidelines: diagnosis of tuberculosis in adults and children. Clin Infect Dis. 2017;64:111-115.

38. Alcántara-Payawal DE, Matsumura M, Shiratori $Y$, Okudaira T, González R, López RA, et al. Direct detection of mycobacterium tuberculosis using polymerase chain reaction assay among patients with hepatic granuloma. J Hepatol. 1997;27:620-627.

39. Nahid P, Dorman SE, Alipanah N, Barry PM, Brozek JL, Cattamanchi A, et al. Official American Thoracic Society/Centers for Disease Control and Prevention/Infectious Diseases Society of America Clinical Practice Guidelines: treatment of drug-susceptible tuberculosis. Clin Infect Dis. 2016:63:e147-e195.

40. Del Campo-Rodríguez LE, Sifuentes-Osornio J. Opportunistic infections in the acquired immunodeficiency syndrome: the history in Mexico 20 years after the beginning of the epidemic. Rev Invest Clin. 2004:56:169-180.

41. Sonderup MW, Wainwright HC. human immunodeficiency virus infection, antiretroviral therapy, and liver pathology. Gastroenterol Clin North Am. 2017; $46: 327-343$.

42. Poles MA, Dieterich DT, Schwarz ED, Weinshel EH, Lew EA, Lew R et al. Liver biopsy findings in 501 patients infected with human immunodeficiency virus (HIV). J Acquir Immune Defic Syndr Hum Retrovirol. 1996:11:170-177.

43. Lizardi-Cervera J, Soto-Ramírez LE, Poo JL, Uribe M. Hepatobiliary diseases in patients with human immunodeficiency virus (HIV) treated with non-highly active anti-retroviral therapy: frequency and clinical manifestations. Ann Hepatol. 2005:4:188-191.

44. Wilson EM, Rosenthal ES, Kattakuzhy S, Tang L, Kottilil S. Clinical laboratory testing in the era of directly acting antiviral therapies for hepatitis C. Clin Microbiol Rev. 2017:30:23-42.

45. Piñeirúa $A$, Sierra-Madero J, Cahn $P$, Guevara-Palmero RN, Martínez-Buitrago E, Young B, et al. The HIV care continuum in Latin America: challenges and opportunities. Lancet Infect Dis. 2015;15:833-839.

46. Araujo-Meléndez J, Sifuentes-Osornio J, Bobadilla-Del Valle JM, Aguilar-Cruz A, Torres-Ángeles O, Ramírez-González JL, et al. What do we know about $Q$ fever in Mexico? Rev Invest Clin. 2012;64:541-5.

47. Dean AS, Crump L, Greter H, Schelling E, Zinsstag J. Global burden of human brucellosis: a systematic review of disease frequency. PLoS Negl Trop Dis. 2012;6:e1865

48. Hernández-Mora G, Ruiz-Villalobos N, Bonilla-Montoya R, Romero-Zúniga JJ, Jiménez-Arias J, González-Barrientos R, et al. Epidemiology of bovine brucellosis in Costa Rica: lessons learned from failures in the control of the disease. PLoS One. 2017;12:e0182380. 
49. Borba MR, Stevenson MA, Goncalves VS, Neto JS, Ferreira F, Amaku M, et al. Prevalence and risk-mapping of bovine brucellosis in Maranhão State, Brazil. Prev Vet Med. 2012;110:169-176.

50. Poulsen KP, Hutchins FT, McNulty CM, Tremblay M, Zabala C Barragan V, et al. Brucellosis in dairy cattle and goats in northern Ecuador. Am J Trop Med Hyg. 2014;90:712-715.

51. Costa PS, Brigatte ME, Greco DB. Questing one Brazilian query: reporting 16 cases of $Q$ fever from Minas Gerais, Brazil. Rev Inst Med Trop Sao Paulo. 2006;48:5-9.

52. Geha R, Peters M, Gill RM, Dhaliwal G. Histology rings true. N Engl J Med. 2017;376:869-874

53. Kersh GJ. Antimicrobial therapies for $Q$ fever. Expert Rev Anti Infect Ther. 2013;11:1207-1214.

54. Rubach MP, Halliday JE, Cleaveland S, Crump JA. Brucellosis in low-income and middle-income countries. Curr Opin Infect Dis. 2013;26:404-412.

55. Young EJ, Hasanjani-Roushan MR, Shafae S, Genta RM, Taylor SL. Liver histology of acute brucellosis caused by Brucella melitensis. Hum Pathol. 2014;45:2023-2028.

56. Pappas PG, Kauffman CA, Andes DR, Clancy CJ, Marr KA, Ostrosky-Zeichner $\mathrm{L}$, et al. Clinical practice guideline for the management of candidiasis: 2016 update by the Infectious Diseases Society of America. Clin Infect Dis. 2016;62:e1-e50.

57. Rammaert B, Desjardins A, Lortholary O. New insights into hepatosplenic candidosis, a manifestation of chronic disseminated candidosis. Mycoses. 2012:55:e74-e84

58. Fortún J, Meije Y, Fresco G, Moreno S. Aspergillosis. Clinical forms and treatment. Enferm Infecc Microbiol Clin. 2012;30:201-208.

59. Chen L, Liu Y, Wang W, Liu K. Adrenal and hepatic aspergillosis in an immunocompetent patient. Infect Dis (Lond). 2015;47:428-432.

60. Piratvisuth T, Siripaitoon P, Sriplug H, Ovartlarnporn B. Findings and benefit of liver biopsies in 46 patients infected with human immunodeficiency virus. J Gastroenterol Hepatol. 1999;14:146-149.

61. Sifuentes-Osornio J, Corzo-León DE, Ponce-De León LA. Epidemiology of invasive fungal infections in Latin America. Curr Fungal Infect Rep. 2012;6:23-34

62. Galgiani JN, Ampel NM, Blair JE, Catanzaro A, Geertsma F, Hoover SE, et al. 2016 Infectious Diseases Society of America (IDSA) clinical practice guideline for the treatment of coccidioidomycosis. Clin Infect Dis. 2016:63:e112-e146.

63. Heninger E, Hogan LH, Karman J, Macvilay S, Hill B, Woods JP, et al. Characterization of the histoplasma capsulatum-induced granuloma. $\mathrm{J}$ Immunol. 2006;177:3303-3313.

64. Sifuentes-Osornio J, Ponce-De León A. Diagnosis and treatment of non-European fungal infections. Curr Fungal Infect Rep. 2014;8:343-352.

65. Kothadia JP, Kone V, Giashuddin S. Granulomatous hepatitis: a rare primary manifestation of disseminated histoplasmosis in a renal transplant recipient. J Gastrointestin Liver Dis. 2017;26:114.

66. Niembro-Ortega MD, Martínez-Gamboa RA, Crabtree-Ramírez B, Martínez-Ayala P, Rangel-Cordero A, Velázquez-Zavala, NG, et al. PCR and urine antigen for diagnosis of disseminated histoplasmosis in AIDS patients. Poster session presented at: Conference on retroviruses and opportunistic infections. EE. UU.: 2018.

67. Ozaras R, Tahan V, Mert A, Uraz S, Kanat M, Tabak F, et al. The prevalence of hepatic granulomas in chronic hepatitis C. J Clin Gastroenterol. 2004;38:449-452.

68. Snyder N, Martínez JG, Xiao SY. Chronic hepatitis C is a common associated with hepatic granulomas. World J Gastroenterol. 2008;14: 6366-6369.

69. Tahan V, Ozaras R, Lacevic N, Ozden E, Yemisen M, Ozdogan O, et al. Prevalence of hepatic granulomas in chronic hepatitis B. Dig Dis Sci. 2004;49:1575-1577.

70. Harada K, Minato H, Hiramatsu K, Nakanuma Y. Epithelioid cell granulomas in chronic hepatitis $\mathrm{C}$ : immunohistochemical character and histological marker of favourable response to interferon-alpha therapy. Histopathology. 1998;33:216-221.

71. Noya O, Katz N, Pointier JP, Theron A, De Noya BA. Schistosomiasis in America. En: Franco-Paredes C, Santos-Preciado Jl, editores. Neglected tropical diseases - Latin America and the Caribbean. Austria: Springer; 2015.

72. Francolugo-Vélez VA ZAJ. Infección del tracto urinario por Schistosoma haematobioum. Un caso en Cuernavaca, Morelos, México. Rev Mex Urol. 2010;70:187-192.

73. Avalos D, Nimeh W, Varela G. A case of schistosomiasis haematobium diagnosed in Mexico in a patient from Egypt. Rev Inst Salubr Enferm Trop. 1963;23:45-54

74. Kadin ME, Donaldson SS, Dorfman RF. Isolated granulomas in Hodgkin's disease. N Engl J Med. 1970;283:859-861.

75. Ruiz-Argüelles GJ, Mercado-Díaz MA, Ponce-De León S, Ponce-De León S, Pérez-Tamayo R. Studies on lymphomata. III. Lymphomata, granulomata and tuberculosis. Cancer. 1983;52:258-262.

76. Carrillo-Pérez DL, Apodaca-Cháveza El, Carrillo-Maravilla E, Mejía-Ávila M, Hernández-Oropeza JL, Reyes E, et al. Sarcoidosis: a single hospital-based study in a 24-year period. Rev Invest Clin. 2015; 67:33-38.

77. Purriel $P$, Navarrete E. Epidemiology of sarcoidosis in Uruguay and other countries of Latin America. Am Rev Respir Dis. 1961;84:155-161.

78. Iwai K, Oka H. Sarcoidosis. Report of ten autopsy cases in Japan. Am Rev Respir Dis. 1964;90:612-622.

79. Judson MA. Extrapulmonary sarcoidosis. Semin Respir Crit Care Med. 2007;28:83-101.

80. Syed U, Alkhawam H, Bakhit M, Companioni RA, Walfish A. Hepatic sarcoidosis: pathogenesis, clinical context, and treatment options. Scand J Gastroenterol. 2016;51:1025-1030.

81. Ishak KG, Zimmerman HJ. Drug-induced and toxic granulomatous hepatitis. Baillieres Clin Gastroenterol. 1988;2:463-480.

82. Zhang X, Ouyang J, Thung SN. Histopathologic manifestations of drug-induced hepatotoxicity. Clin Liver Dis. 2013;17:547-564. 


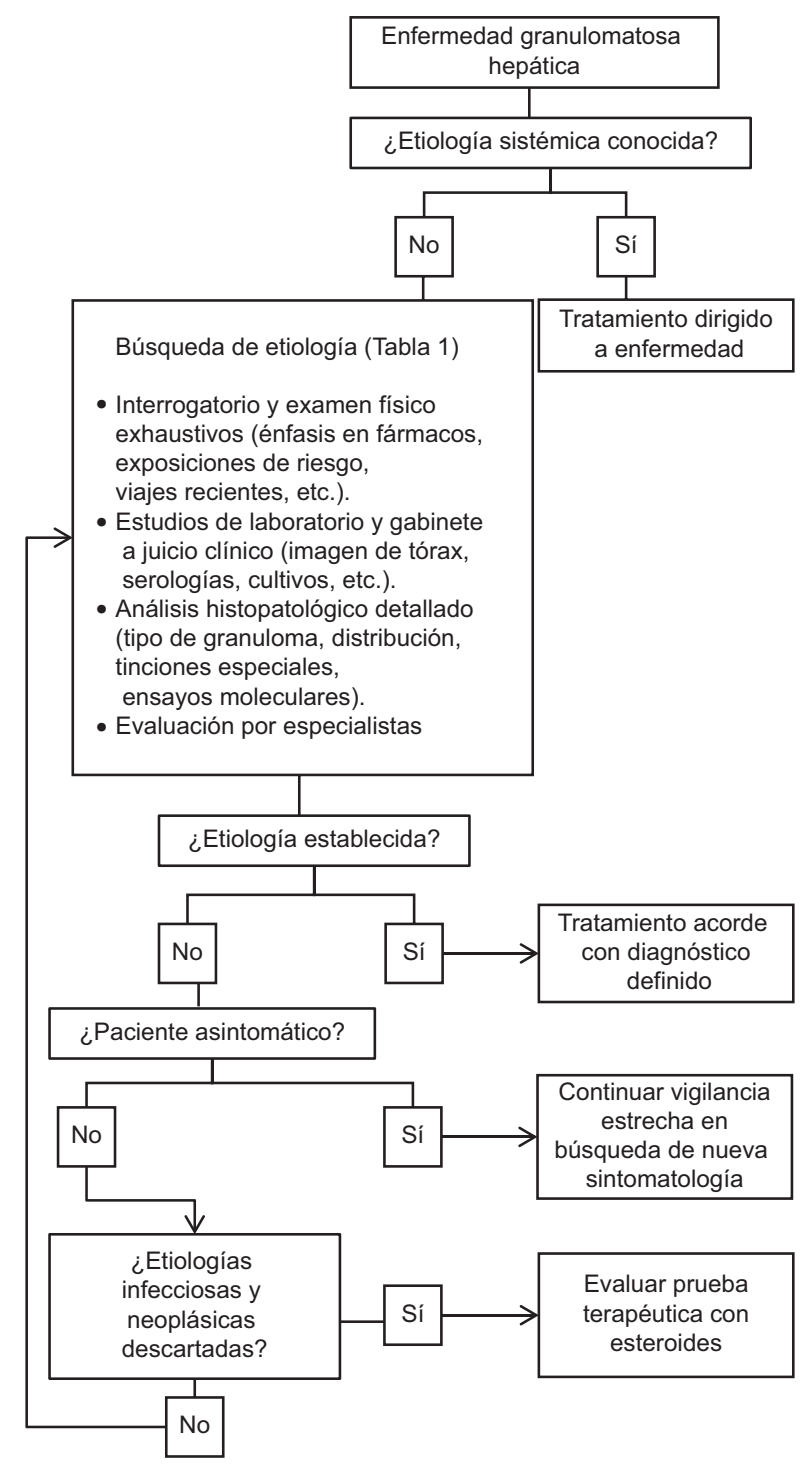

Algoritmo 1. Estudio de la enfermedad granulomatosa hepática. 